\title{
MODELAGEM DAS DISTRIBUIÇÕES DAS
}

TAXAS DE RETORNO DOS

ÍNDICES IBOVESPA E S\&P500

\section{MODELING THE DISTRIBUTIONS OF THE IBOVESPA AND S\&P5OO RATES OF RETURN}

\section{FRANCISCO HENRIQUE FIGUEIREDO DE CASTRO JUNIOR}

Doutor em Administração pela Universidade de São Paulo (USP). Professor pesquisador do Programa de Mestrado em Ciências Contábeis do Centro Universitário FECAP. Avenida Liberdade, 532, Liberdade - São Paulo - SP - CEP $01502-001$ E-mail: henrique.castro@fecap.br

\section{HÉBER PESSOA DA SILVEIRA}

Doutor em Administração pela Universidade de São Paulo (USP). Professor colaborador do Programa de Mestrado em Ciências Contábeis do Centro Universitário FECAP Avenida Liberdade, 532, Liberdade - São Paulo - SP - CEP $01502-001$ E-mail: heberpessoa@yahoo.com.br 


\section{RESUMO}

Discussões acerca da distribuição de retornos de índices de ações têm sido recorrentes na literatura financeira, especialmente por seu uso em modelos que adotam a normalidade desses retornos como premissa. Seguindo a linha dos trabalhos de Ingersoll Jr. (1987) e Hamada e Valdez (2004), analisou-se a distribuição empírica dos retornos do Ibovespa e S\&P500 no período de I986 a 2006. Assim como reportado em trabalhos anteriores, para ambos os índices, ficou evidenciado que a suposição de normalidade pode ser altamente questionável, pois outras distribuições teóricas da família elíptica apresentaram um nível superior de aderência aos dados empíricos. Os retornos dos índices de ações tanto do Brasil quanto dos Estados Unidos apresentaram leptocurtose, e no caso do Brasil verificou-se a presença até mesmo de assimetria, de forma que apenas distribuições que lidam com tais parâmetros de modo menos restritivo, a exemplo da Logística e Log-Logística, puderam apresentar melhor aderência aos dados observados.

\section{PALAVRAS-CHAVE}

Normalidade; Taxas de retorno; Distribuições elípticas; CAPM; Máxima verossimilhança.

\section{ABSTRACT}

Stock market indices' rate of return distributions is a recurrent theme in financial literature, especially because some models require the normality assumption as premise. Following the works of Ingersoll Jr. (I987) and Hamada \& Valdez (2004), the empirical distribution of the Ibovespa and S\&P5O0 rates of returns from 1986 to 2006 was analyzed. As reported by some others authors, for both indices, the results evidenced that the normality assumption can be highly questionable, because other theoretical distributions from the elliptical family fitted the data better. The rates of return for the Brazilian and American indices 
showed leptokurtosis, and, in the Brazilian case, asymmetric shape, so that only distributions that deal with these parameters in a less restrictive way, as the Logistic or Log-logistic distributions, can show a better fit to the observed data.

\section{KEYWORDS}

Normality; Rate of return; Elliptical distributions; CAPM; Maximum likelihood.

\section{INTRODUÇ ÃO}

Um tema recorrente na literatura de finanças trata da distribuição dos retornos de ações e índices. Modelos como o Capital Asset Pricing Model (CAPM) se utilizam, em algumas derivações, da pressuposição, nem sempre explícita, de normalidade nos retornos dos ativos. Algumas restrições contidas no CAPM foram evidenciadas já por seus criadores. O artigo seminal de Sharpe (i964, p. 434) afirma, por exemplo, que algumas de suas pressuposições acerca do comportamento dos agentes de mercado e de suas expectativas podiam ser altamente restritivas e não realistas, o que também parece ocorrer em relação à inicialmente não comentada pressuposição acerca da normalidade dos retornos.

Buscando analisar a viabilidade do modelo CAPM mesmo na ausência de algumas das pressuposições adotadas por seus criadores, diversos autores têm tentado abordagens alternativas na derivação do mesmo. Cochrane (2005) deriva o CAPM por meio de quatro formas distintas, sendo os pressupostos em cada uma delas: i. a função de utilidade dos agentes é quadrática; ii. a função de utilidade dos agentes é exponencial, de dois períodos e os retornos se distribuem normalmente; iii. um horizonte infinito, utilidade quadrática e retornos independentes e identicamente distribuídos; e iv. uma função de utilidade logarítmica para os agentes econômicos.

O modelo de derivação por função exponencial, e que assume a normalidade dos retornos, termina por ser provavelmente o mais utilizado. Cochrane (2005, p. I52) afirma que as diversas formas de derivação podem ser utilizadas, desde que as hipóteses utilizadas sejam mantidas. Segundo ele, o CAPM não requer a normalidade da distribuição da taxa de retorno desde que a função de utilidade do agente econômico seja do tipo quadrática. Pode-se, entretanto, argumentar que o uso dessa função de utilidade quadrática é frequentemente criticado por autores, para quem essa função possui inadequações do ponto de vista econô- 
mico. Segundo Gollier (200I, p. 27), a função de utilidade quadrática tem sido reportada na literatura apenas em virtude da facilidade de manipulação, dado que algumas incoerências de sua aplicação em economia são evidentes, a exemplo do seu domínio restrito para a modelagem econômica, pois a função de utilidade quadrática possui um ponto de ótimo a partir do qual mais riqueza seria considerada indesejável pelo agente econômico. Outro aspecto de contradição dessa função de utilidade é o aumento do coeficiente de aversão ao risco do agente à medida que sua riqueza cresce. Esse fato é incompatível com observações empíricas, como em Rosenzweigh e Binswanger (1993); e em Blake (1996), que reportam diminuição no prêmio pelo risco com o aumento da riqueza. Nas palavras de Gollier (200I, p. 27) não há razões óbvias para se acreditar que a função de utilidade quadrática represente adequadamente a atitude do agente frente ao risco no mundo real.

O uso da função de utilidade exponencial em adição à normalidade dos retornos é citado em Cochrane (2005, p. 154) como tendo uma formulação analítica conveniente, pois se adapta a modelos mais complexos como os de Grossman e Stiglitz (I980) que consideram a existência de mercados incompletos e assimetria de informação entre diversos agentes. Essa limitação da função de utilidade a ser assumida com mínima perda em relação ao comportamento efetivamente esperado pelos agentes leva a que se insista na derivação do modelo assumindose a função de utilidade exponencial e normalidade dos retornos. Um problema surge, entretanto, nesse momento justamente quando os dados não se comportam exatamente como assumido pelo modelo, ou seja, quando os retornos observados insistem em não se apresentar como normalmente distribuídos.

A inadequação da suposição de normalidade nos dados de retorno tem sido frequentemente reportada. Já em I965, Fama verificou que os retornos diários da bolsa de valores de Nova York apresentavam curtose superior ao observado em uma distribuição normal. Campbell, Lo e MacKinlay (I997), mais de trinta anos depois, reforçam o estudo afirmando que a estimação da curtose em amostras de retornos diários na bolsa americana é mais alta que o esperado sob a suposição de normalidade. Segundo Campbell, Lo e MacKinlay (I997, p. I7), estimações feitas a partir de séries da taxa de retornos diários de ações americanas mostram excesso de curtose tanto para ações individuais como para índices de ações, indicando que a taxa de retorno possui mais massa nas caudas que o previsto por uma distribuição normal.

No Brasil, entre outros autores, Torres, Bonomo e Fernandes (2000) chegaram a resultados similares. Em uma carteira formada por 62 ações entre as mais líquidas da Bovespa no período de 1986 a I998, foi verificado que a curtose dos retornos ficou, em todos os casos, entre sete e oito, valores bem superiores 
ao esperado para uma distribuição normal. Os três testes utilizados pelos autores (Jarque-Bera, regressão dos quantis e índice de caudas) foram unânimes em rejeitar a hipótese nula de normalidade, mostrando, além da leptocurtose das séries, que a taxa de retornos diários apresentou assimetria positiva, enquanto a taxa de retornos semanais apresentou assimetria negativa, contrariando outra característica fundamental da normal: a simetria.

Essa fuga de normalidade leva a perdas informacionais e a frequentes especificações inadequadas de modelos mais complexos que se utilizam dessa suposição, como o CAPM ou o modelo de Black \& Scholes de apreçamento de opções. Essa perda informacional tem motivado estudos a respeito do impacto nos modelos financeiros mais utilizados quando a pressuposição de normalidade não pode ser mantida. Badrinath e Chatterjee (I988), além de fornecerem novas evidências acerca das características de distribuições empíricas, fornecem formas funcionais de distribuições que aperfeiçoam as estratégias de formação de carteira de ativos e sugerem modelos de determinação de preço que incorporam momentos de ordem superior à esperança e variância comumente utilizadas.

\section{REFERENCIAL TEÓRICO}

\subsection{FAMÍLIA DE DISTRIBUIÇÕES ELÍPTICAS SIMÉTRICAS}

Analisando toda uma classe de distribuições, Ingersoll Jr. (I987) afirma que a normal multivariada não é a única distribuição que torna a análise de médiavariância consistente com o princípio da maximização da utilidade esperada. Segundo o autor, toda a família das distribuições elípticas se mostra adequada a análises por média-variância. Um conjunto de variáveis aleatórias pode ser considerado elipticamente distribuído se sua função de densidade é dada pela fórmula:

$$
f(x)=|\Omega|^{-\mathrm{I} / 2} g\left[(x-\mu)^{\mathrm{T}} \Omega^{-\mathrm{I}}(x-\mu) ; n\right],
$$

tal que $\Omega$ é a matriz de dispersão positiva definida, $\mu$ é o vetor de medianas e $\mathrm{g}(\cdot)$ é uma função univariada com parâmetro $n$. Se as variâncias da distribuição multivariada forem finitas, será proporcional à matriz de covariâncias. Se as esperanças existirem, o vetor $\mu$ será também o vetor de médias. Essas preocupações quanto à existência dos momentos se deve ao fato da distribuição Cauchy (um caso particular da distribuição $t$ de Student multivariada) fazer parte da família das distribuições elípticas e não ter nenhum momento definido. 
Seguindo linha de raciocínio similar a Ingersoll Jr. (1987), Hamada e Valdez (2004) sugerem uma prova alternativa do CAPM e de modelos de apreçamento de opções baseados em distribuições elípticas. A prova, entretanto, é menos restritiva em relação às pressuposições acerca da curtose dos dados. Contudo, ainda é exigido que a distribuição da taxa de retornos seja simétrica.

Se tais estudos despertam interesses no mercado americano, no Brasil, onde as observações extremas que levam as distribuições das taxas de retorno a apresentarem "caudas pesadas" são ainda mais frequentes, a compreensão e o estudo de tais resultados se mostram um campo promissor para a pesquisa em finanças.

\subsubsection{Distribuição normal}

Segundo Branco e Arellano-Valle (2004), a classe de distribuições elípticas surge como uma extensão das distribuições normais. Portanto, a fim de introduzir as distribuições que fazem parte dessa família, será dada prioridade à mais conhecida delas.

Uma variável aleatória X é dita normalmente distribuída se sua função densidade de probabilidade é:

$$
f_{X}(x, \theta)=\frac{\mathrm{I}}{\sqrt{2 \pi \sigma}} \exp \left\{-\frac{\mathrm{I}}{2} \frac{(x-\mu)^{2}}{\sigma^{2}}\right\}, \theta \equiv\left(\mu, \sigma^{2}\right) \in R \times R_{+}, x \in R .
$$

A notação largamente conhecida é de que

$$
X \sim N\left(\mu, \sigma^{2}\right),
$$

e a distribuição normal padrão,

$$
N(\mathrm{O}, \mathrm{I})
$$

é dada por:

$$
f_{z}(z, \theta)=\frac{\mathrm{I}}{\sqrt{2 \pi}} \exp \left\{-\frac{\mathrm{I}}{2} z^{2}\right\}, \theta \equiv(\mathrm{O}, \mathrm{I}), z \in R
$$




\subsubsection{Distribuição $t$ de Student}

Uma variável aleatória $Y$ é distribuída conforme uma $t$ de Student padrão,

$$
Y \sim t(0, I, v)
$$

se sua função densidade de probabilidade for dada por:

$$
f(\gamma, \theta)=\frac{\Gamma\left[\frac{v+\mathrm{I}}{2}\right]}{\Gamma\left[\frac{v}{2}\right] \sqrt{v \pi}}\left[\frac{v+\gamma^{2}}{v}\right]^{-\frac{v+1}{2}}, \theta \equiv(0, \mathrm{I}, v), \gamma \in R .
$$

tal que

$$
\Gamma(a)=\int_{0}^{\infty} b^{a} x^{a-1} \exp \{-b x\} d x
$$

é a função gama.

A distribuição normal padrão é um caso particular da distribuição $t$ de Student padrão, sendo seu grau de liberdade $v=\infty$. Outra distribuição que apresenta relação direta com a $t$ de Student padrão é a Cauchy: uma Cauchy é uma $t$ de Student com I grau de liberdade.

A forma geral da distribuição $t$ de Student é a que segue:

$$
f(\gamma, \theta)=\frac{\Gamma\left[\frac{v+\mathrm{I}}{2}\right]\left(\sigma^{2} v \pi\right)^{-1 / 2}}{\Gamma\left[\frac{v}{2}\right]}\left[\mathrm{I}+\frac{(\gamma-\mu)^{2}}{v \sigma^{2}}\right]^{-\frac{v+1}{2}}, \theta \equiv\left(\mu, \sigma^{2}, v\right) \in R \times R_{+}, \gamma \in R .
$$

\subsubsection{Distribuição logística}

Uma variável aleatória $X$ é distribuída conforme uma logística, $X \sim \operatorname{Lg}(\alpha, \beta)$ se sua função densidade de probabilidade for dada por: 


$$
f(x, \theta)=\frac{\exp \left\{-\left(\frac{x-\alpha}{\beta}\right)\right\}}{\beta\left(I+\exp \left\{-\left(\frac{x-\alpha}{\beta}\right)\right\}\right)^{2}}, \theta \equiv(\alpha, \beta) \in R \times R_{+}, x \in R .
$$

\subsubsection{Outras distribuições da família das elípticas}

Outras distribuições da família das distribuições elípticas são a Bessel, Laplace, Pearson do tipo II, Pearson do tipo VII, dentre outras. Para maiores detalhes sobre essas distribuições, como suas funções densidades de probabilidade, funções características e relações com outras distribuições, recomendam-se os textos de Gupta e Varga (I993), Spanos (I999) e Hamada e Valdez (2004).

A Figura I permite comparar visualmente a diferença entre algumas distribuições de probabilidade da família das elípticas. Estão plotadas as distribuições logística com parâmetros

$$
\left(0 ; \frac{\sqrt{3}}{\pi}\right) \text {, }
$$

a normal padronizada e $t$ de Student com 2 graus de liberdade.

\section{FigurA I}

FUNÇÕES DENSIDADE DE PROBABILIDADE DA FAMÍLIA ELÍPTICA

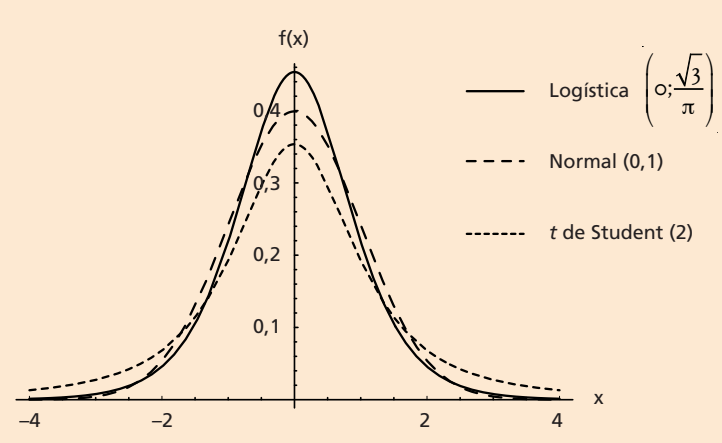

Fonte: Elaborada pelos autores. 


\subsection{DISTRIBUIÇÕES DE PROBABILIDADES ASSIMÉTRICAS}

Dados empíricos de taxas de retorno podem ser assimetricamente distribuídos. Algumas distribuições servem a esse princípio, como as distribuições Burr do tipo III, Weibull ou Beta Generalizada do tipo 2 (BG2). Esta última distribuição foi introduzida na literatura de finanças por Bookstaber e McDonald (I987) para descrever o comportamento da taxa de retorno de ações. A grande vantagem dessa distribuição está em sua flexibilidade em lidar com assimetria e curtose. Distribuições como a log-normal, log-t, log-Cauchy e log-logística são casos particulares da BG2.

A função densidade de probabilidade da BG2 é dada pela seguinte expressão:

$$
f(x, \theta)=\frac{\alpha(x-\gamma)^{\alpha p-\mathrm{I}}}{\beta^{\alpha p} B(p, q)\left[\mathrm{I}+\left(\frac{x-\gamma}{\beta}\right)^{\alpha}\right]^{p+q}}, \theta \equiv(\alpha, \beta, p, q) \in R \times R_{+},(x-\gamma)>0,
$$

tal que $B(p, q)$ é a distribuição beta com parâmetros $(p, q)$.

A distribuição log-logística, caso particular da BG2, é descrita como:

$$
L L(\alpha, \beta) \equiv B G 2(\alpha, \beta, \mathrm{I}, \mathrm{I})=\frac{\alpha\left(\frac{x-\gamma}{\beta}\right)^{\alpha-\mathrm{I}}}{b\left[\mathrm{I}+\left(\frac{x-\gamma}{\beta}\right)^{\alpha}\right]^{2}} .
$$

O domínio da distribuição log-logística está definido no intervalo $[\gamma,+\infty]$. É uma distribuição claramente assimétrica à direita, e serve para modelar taxas de retorno que são positivamente assimétricas.

A Figura 2 permite comparar visualmente a diferença entre a distribuição normal padronizada e a log-logística $L L(7,4,-4)$. 
- MODELAGEM DAS DISTRIBUIÇÕES DAS TAXAS DE RETORNO DOS ÍNDICES IBOVESPA E S\&P500 •

\section{FIGURA 2}

\section{FUNÇÕES DENSIDADE DE PROBABILIDADE NORMAL PADRÃO} E LOG-LOGÍSTICA

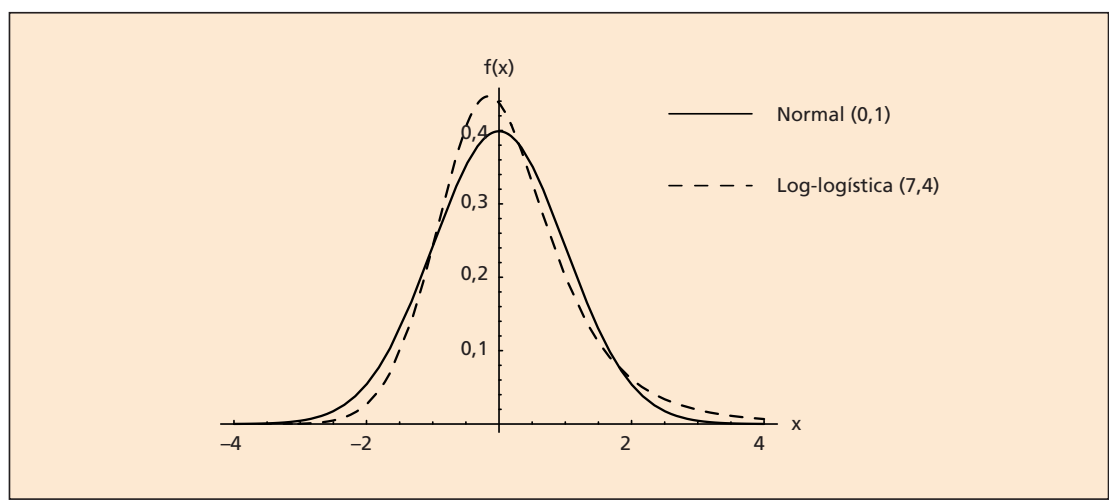

Fonte: Elaborada pelos autores.

\section{PROCEDIMENTO METODOLÓGICO}

A fim de estimar a função de densidade univariada das observações das taxas de retorno dos índices Ibovespa e S\&P500, usar-se-á o Método da Máxima Verossimilhança (MMV). A função de verossimilhança foi proposta inicialmente por Fisher (I9I2). Por esse método, conforme Spanos (I999), é possível estimar um modelo estatístico $S \equiv(\Phi, \mathbf{X})$ que descreva probabilisticamente o mecanismo estocástico que deu origem aos dados observados $\mathbf{x} \equiv\left(x_{\mathrm{I}}, x_{2}, \ldots, x_{n}\right)$.

O modelo estatístico é descrito como um modelo de probabilidade na forma

$$
\Phi=f\{f(x, \theta), \theta \in \Theta, x \in R\}
$$

e um modelo amostral dado por

$$
X \equiv\left(X_{\mathrm{I}}, X_{2}, \ldots, X_{n}\right)
$$

A distribuição conjunta das variáveis aleatórias que compõem esta amostra é

$$
D\left(X_{\mathrm{I}}, X_{2}, \ldots, X_{n} ; \theta\right)
$$


Essa distribuição $D$ é uma distribuição da amostra, e não deve ser confundida com a distribuição da realização amostral, que é o interesse da estimação.

O MMV, portanto, entende o vetor $\mathbf{x}$ como uma realização amostral, e define a função de verossimilhança como proporcional à distribuição da amostra, mas interpretada como uma função de $\theta$ :

$$
\varsigma\left(\theta, x_{1}, x_{2}, \ldots, x_{n}\right) \infty D\left(X_{1}, X_{2}, \ldots, X_{n} ; \theta\right)
$$

Segundo Mittelhammer, Judge e Miller (2000), a essência do MMV é encontrar o estimador de máxima verossimilhança $\hat{\theta}$ de $\theta$ para a função densidade de probabilidade de forma que os dados observados se ajustem a ela. Assim, a estimação por máxima verossimilhança se dá com a resolução do programa:

$$
\varsigma(\hat{\theta} ; \mathbf{x})=\max _{\theta \in \Phi} \varsigma(\theta ; \mathbf{x})
$$

Conforme Spanos (I999), apesar da existência de casos patológicos que podem ser verificados em Gourieroux e Monfort (I995), na grande maioria dos casos, o programa descrito na equação anterior existirá e terá solução única. Para encontrá-la, basta diferenciar $\varsigma(\theta, \mathbf{x})$, e o Estimador de Máxima Verossimilhança (EMV) será encontrado por meio da condição de primeira ordem:

$$
\frac{d \varsigma(\theta, \mathbf{x})}{d \theta}=g\left(\hat{\theta}_{M} V\right)=0
$$

dado que

$$
\left.\frac{d^{2} \varsigma(\theta, \mathbf{x})}{d \theta^{2}}\right|_{\theta=\hat{\theta}_{E M V}} .
$$

Dentre os modelos estatísticos a serem estimados, estão especialmente as funções densidades de probabilidade da família das elípticas. Serão utilizadas a distribuição Normal, a distribuição Logística, a distribuição Log-logística e a distribuição $t$ de Student. Como critério de ordenação da qualidade do ajuste realizado para cada distribuição, será usado o teste do Chi-quadrado. O teste é definido como: 


$$
\chi^{2}=\sum_{i=\mathrm{I}}^{k} \frac{\left(O_{i}-E_{i}\right)^{2}}{E_{\mathrm{I}}},
$$

tal que $O_{i}$ é a frequência observada da classe i do histograma dos dados observados e $E_{i}$ é a frequência esperada de observações na classe i. A quantidade k é o número de classes do histograma dos dados, e seu valor crítico é calculado para $\mathrm{k}$ - I graus de liberdade.

\section{RESULTADOS E ANÁLISES}

Para a estimação da distribuição amostral pelo método da máxima verossimilhança da taxa de retorno diária do Ibovespa foi usado o período de 2 de janeiro de 1986 a I2 de abril de 2006 . Foi usada a série após o ajuste pela inflação e também na sua forma original. A fim de comparar os resultados encontrados para o índice nacional, foi usado ainda o índice americano S\&P500, também na sua forma original e ajustado pela inflação. Os resultados encontrados estão apresentados a seguir.

\subsection{IINDICE BOVESPA}

Utilizando-se os dados da série de retornos diários do Ibovespa sem o ajuste para a inflação foi possível estimar a distribuição de probabilidade que melhor aproxima os dados. A partir desse resultado, foi aplicado o teste de $\chi^{2}$ para testar a qualidade do ajuste.

Os resultados estão descritos na Tabela I. Nela, as distribuições estimadas que melhor aproximaram os dados foram ordenadas de acordo com o nível de significância observado. São mostrados também os parâmetros de cada uma das distribuições.

$\mathrm{Na}$ Figura 3 é possível verificar visualmente o ajuste dos dados para as funções log-logística (Figura 3a) e para a função normal (Figura 3b). Fica evidente o ajuste menos perfeito para a distribuição normal. Isso se deve especialmente ao pico mais acentuado na distribuição empírica dos dados, assim como suas caudas mais pesadas.

As distribuições de probabilidade estimadas para a série da taxa de retornos do Ibovespa com correção para a inflação apresentaram problemas no teste do $\chi^{2}$. Isso porque as funções estimadas não apresentaram aderência satisfatória aos dados, sendo todas elas rejeitadas ao nível de 0,05 de significância. A fim de 
contornar esse problema, foram retirados da série os valores extremos que estavam causando problemas na estimação da distribuição. O critério adotado foi o de eliminar da série as observações que estavam a uma distância maior que três desvios-padrão da média da série. Assim, a distribuição de probabilidade que mais se ajustou aos dados foi a Logística. Os resultados podem ser vistos na Tabela 2.

\section{TABELA I}

RESULTADO DO TESTE $\chi^{2}$ PARA A TAXA DE RETORNO DIÁRIA DO IBOVESPA SEM AJUSTE PARA A INFLAÇÃO

\begin{tabular}{c|c|c|c}
\hline RANKING & DISTRIBUIÇÃO DE PROBABILIDADE & PARÂMETROS DA DISTRIBUIÇÃO & SIGNIF. OBSERVADO \\
\hline 1 & Log-logística & $(-1,1280 ; 1,1318 ; 65,159)$ & 0,1137 \\
\hline 2 & Logística & $(0,0040 ; 0,0174)$ & 0,0449 \\
\hline 3 & Normal & $(0,0045 ; 0,0336)$ & 0,0000 \\
\hline
\end{tabular}

Fonte: Dados da pesquisa.

\section{FIgURA 3}

FUNÇÃO LOG-LOGISTICA E NORMAL AJUSTADAS A SÉRIE DA TAXA DE RETORNOS DIÁRIOS DO IBOVESPA

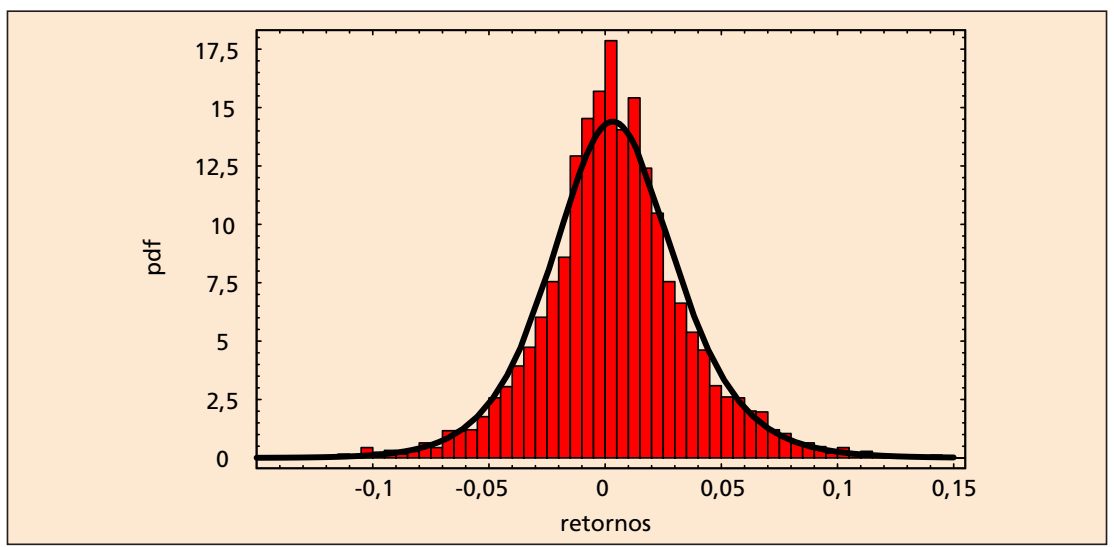

(a) Distribuição Log-logística 
- MODELAGEM DAS DISTRIBUIÇÕES DAS TAXAS DE RETORNO DOS ÍNDICES IBOVESPA E S\&P500 • FRANCISCO HENRIQUE FIGUEIREDO DE CASTRO JUNIOR • HÉBER PESSOA DA SILVEIRA

\section{Figura 3 (CONTINUAÇÃo)}

FUNÇÃO LOG-LOGISTICA E NORMAL AJUSTADAS A SÉRIE DA TAXA DE RETORNOS DIÁRIOS DO IBOVESPA

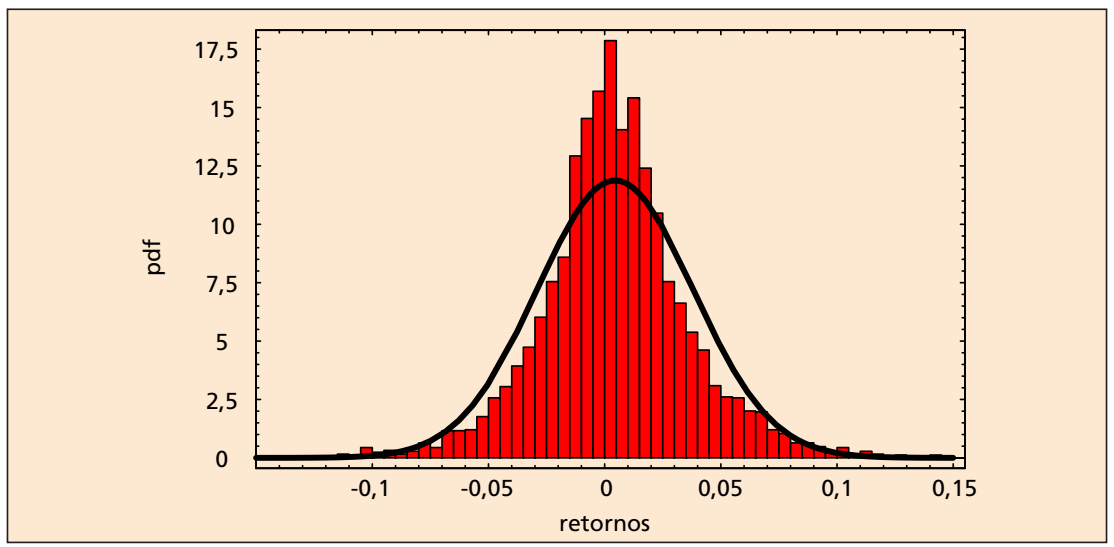

(b) Distribuição Normal

Fonte: Dados da pesquisa.

\section{TABELA 2}

RESULTADO DO TESTE $\chi^{2}$ PARA A TAXA DE RETORNO DIÁRIA DO IBOVESPA APÓS O AJUSTE PARA A INFLAÇÃO

\begin{tabular}{c|c|c|c}
\hline RANKING & DISTRIBUIÇÃO DE PROBABILIDADE & $\begin{array}{c}\text { PARÂMETROS DA } \\
\text { DISTRIBUIÇÃO }\end{array}$ & SIGNIF. OBSERVADO \\
\hline 1 & Logística & $(0,0033 ; 0,0173)$ & 0,1958 \\
\hline 2 & Função erro & $-21,8$ & 0,0000 \\
\hline 3 & Normal & $(0,0035 ; 0,0323)$ & 0,0000 \\
\hline
\end{tabular}

Fonte: Dados da pesquisa.

A Figura 4 apresenta as distribuições logística e normal que melhor se ajustaram à série da taxa de retorno diária do Ibovespa ajustado pela inflação após a eliminação das observações extremas. Mais uma vez é possível ver que a distribuição normal não ajusta tão bem os dados. Nesse caso, a distribuição logística adere melhor aos dados. 


\section{FIGURA 4}

FUNÇÃO LOGÍSTICA E NORMAL AJUSTADAS A SÉRIE DA TAXA DE RETORNOS DIÁRIOS DO IBOVESPA AJUSTADO PELA INFLAÇÃO

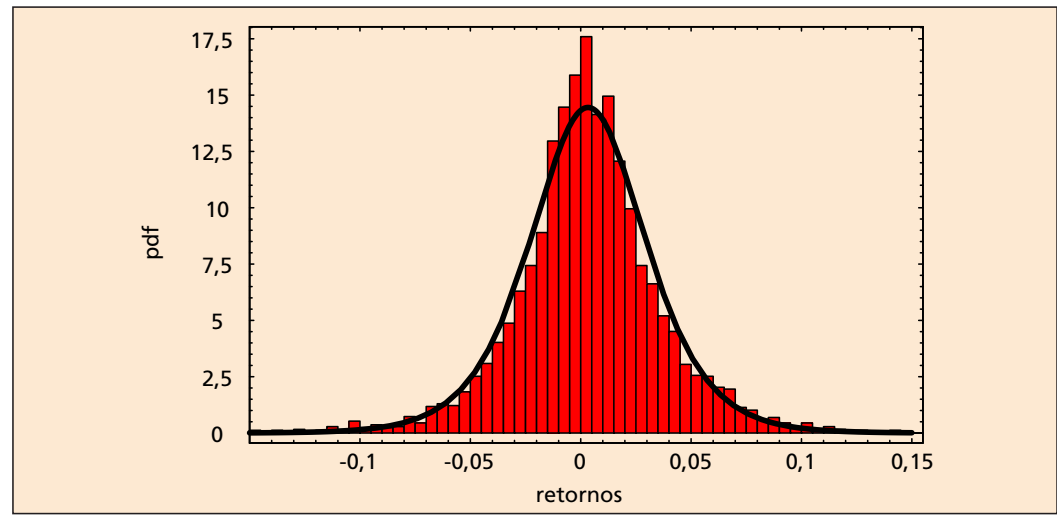

(a) Distribuição Logística

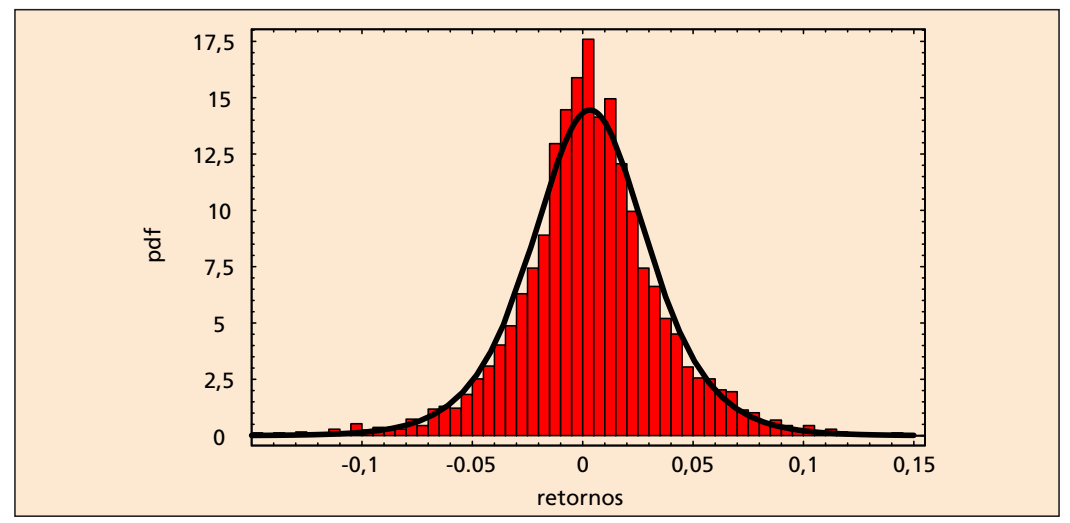

(b) Distribuição Normal

Fonte: Dados da pesquisa.

\section{2 ÍNDICE S\&P500}

A análise da série da taxa de retornos do índice S\&P500 apresentou resultados semelhantes aos do Ibovespa. Utilizando-se primeiramente a série sem o ajuste da inflação na estimação da função densidade, foram obtidos os seguintes resultados para a estimação da distribuição e para o respectivo teste de aderência do tipo $\chi^{2}$, ambos descritos na Tabela 3 . Devido também à presença de observações extremas, foi adotado como critério de eliminação as observações que se situaram a mais de três desvios-padrão da média. 
- MODELAGEM DAS DISTRIBUIÇÕES DAS TAXAS DE RETORNO DOS ÍNDICES IBOVESPA E S\&P500 • FRANCISCO HENRIQUE FIGUEIREDO DE CASTRO JUNIOR • HÉBER PESSOA DA SILVEIRA

\section{TABELA 3}

RESULTADO DO TESTE $\chi^{2}$ PARA A TAXA DE RETORNO DIÁRIA DO S\&P500 SEM O AJUSTE PARA A INFLAÇÃO

\begin{tabular}{c|c|c|c}
\hline RANKING & DISTRIBUIÇÃO DE PROBABILIDADE & $\begin{array}{c}\text { PARÂMETROS DA } \\
\text { DISTRIBUIÇÃO }\end{array}$ & SIGNIF. OBSERVADO \\
\hline 1 & Logística & $(0,0005 ; 0,0050)$ & 0,1385 \\
\hline 2 & Normal & $(0,0004 ; 0,0092)$ & 0,0000 \\
\hline 3 & Função erro & $(77,014)$ & 0,0000 \\
\hline
\end{tabular}

Fonte: Dados da pesquisa.

A visualização dos histogramas na Figura 5 mostra a melhor aderência da distribuição logística. A leptocurtose e a presença de caudas mais pesadas são as razões para que a distribuição normal possua uma menor aderência e, de acordo com o teste do $\chi^{2}$, não represente adequadamente os dados da amostra.

A distribuição logística também aderiu melhor à série da taxa de retornos do índice da S\&P500 ajustado para a inflação após a eliminação de observações extremas. Foi novamente usado o critério de corte de dados acima de três desvios-padrão da média. Os resultados dos testes de $\chi^{2}$ para as distribuições estão na Tabela 4.

A Figura 6 apresenta os histogramas que permitem visualizar a aderência para as distribuições logística e normal estimadas.

\section{FigURA 5}

FUNÇÃO LOGÍSTICA E NORMAL AJUSTADAS A SÉRIE DA TAXA DE RETORNOS DIÁRIOS DO S\&P5O०

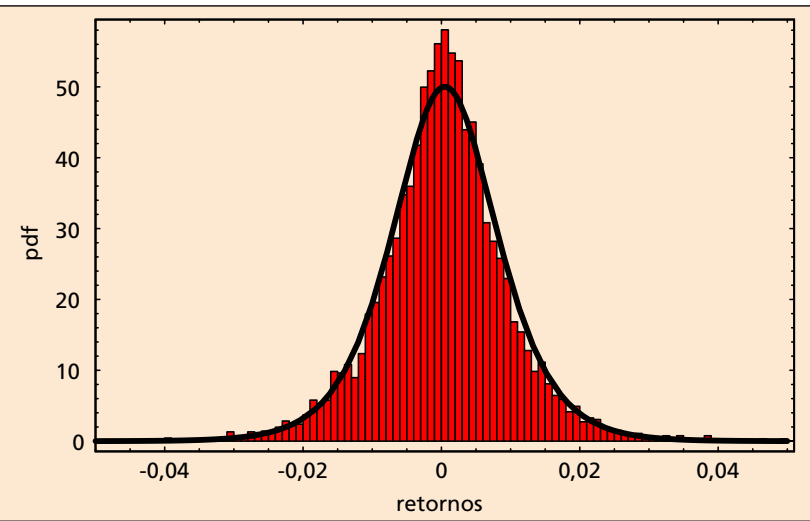

(a) Distribuição Logística 
- RAM - REVISTA DE ADMINISTRAÇÃO MACKENZIE • Volume 10, n. 1, 2009, p. 114-133

\section{Figura 5 (CONTINUAÇÃo)}

FUNÇÃO LOGÍSTICA E NORMAL AJUSTADAS A SÉRIE DA TAXA DE RETORNOS DIÁRIOS DO S\&P500

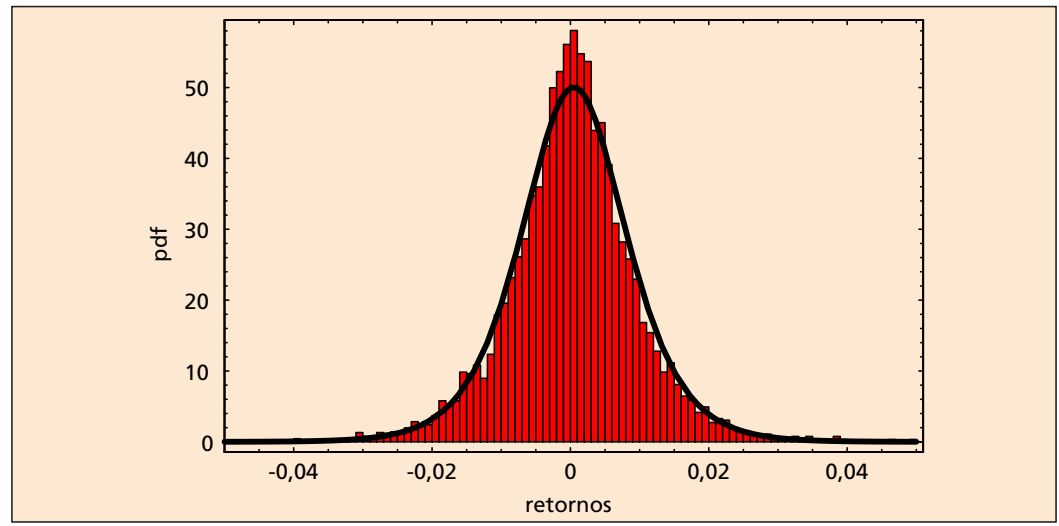

(b) Distribuição Normal

Fonte: Dados da pesquisa.

\section{TABELA 4}

RESULTADO DO TESTE $\chi^{2}$ PARA A TAXA DE RETORNO DIÁRIA DO S\&P500 COM O AJUSTE PARA A INFLAÇÃO

\begin{tabular}{c|c|c|c}
\hline RANKING & DISTRIBUIÇÃO DE PROBABILIDADE & $\begin{array}{c}\text { PARÂMETROS DA } \\
\text { DISTRIBUIÇÃO }\end{array}$ & SIGNIF. OBSERVADO \\
\hline 1 & Logística & $(0,0004 ; 0,0050)$ & 0,1851 \\
\hline 2 & Normal & $(0,0003 ; 0,0092)$ & 0,0000 \\
\hline 3 & Função erro & $(77,052)$ & 0,0000 \\
\hline
\end{tabular}

Fonte: Dados da pesquisa. 
- MODELAGEM DAS DISTRIBUIÇÕES DAS TAXAS DE RETORNO DOS ÍNDICES IBOVESPA E S\&P500 •

\section{Figura 6}

FUNÇÃO LOGÍSTICA E NORMAL AJUSTADAS A SÉRIE DA TAXA DE RETORNOS DIÁRIOS DO S\&P500 COM O AJUSTE PARA INFLAÇÃO

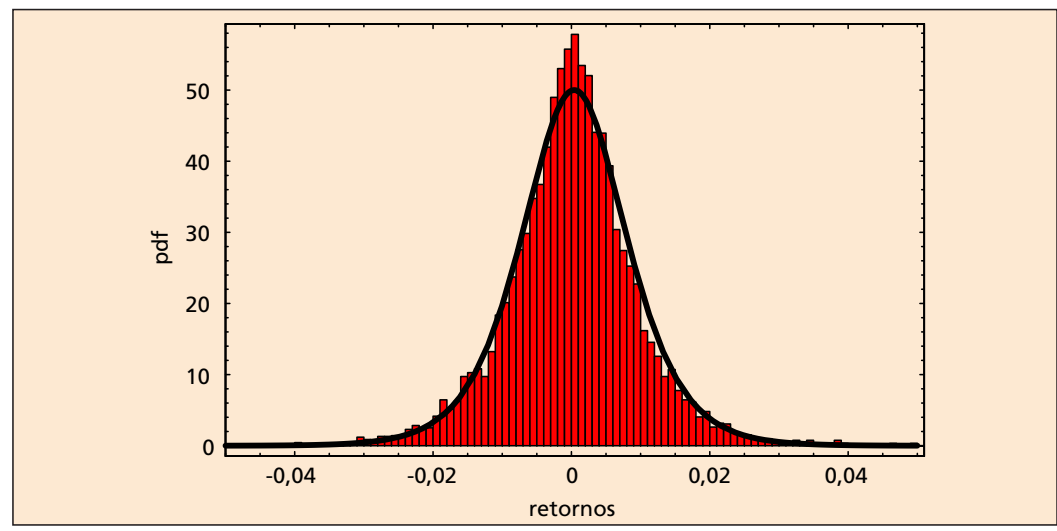

(a) Distribuição Logística

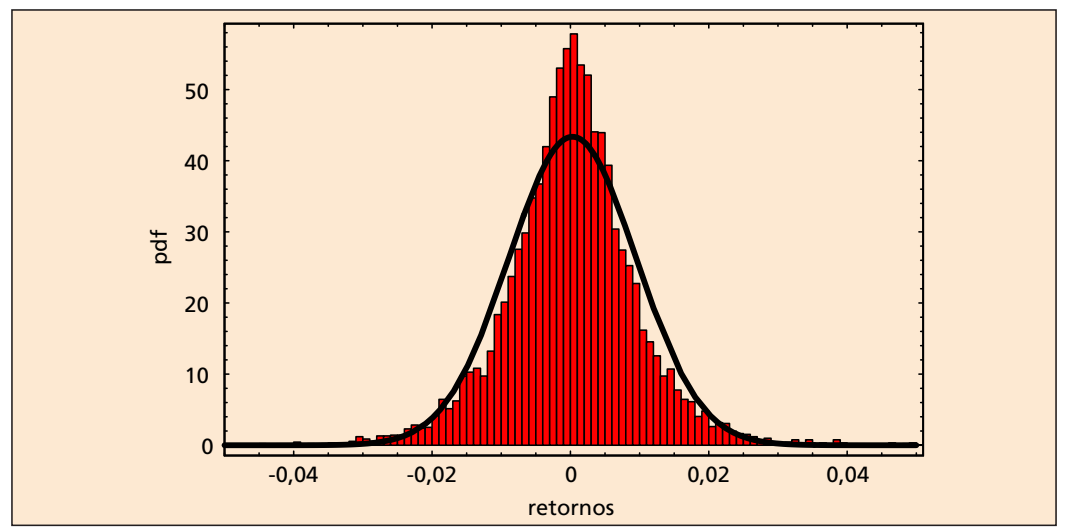

(b) Distribuição Normal

Fonte: Dados da pesquisa.

\section{CONCLUSÕES}

O presente trabalho procurou examinar as características dos retornos diários dos principais índices de ações brasileiro e americano quanto ao formato de sua distribuição no período de janeiro de 1996 a abril de 2006 . Seguindo uma linha de estudos que teve entre seus iniciadores Fama (I965) e Brown e Warner (I985) e que até hoje chama a atenção de pesquisadores, verificou-se que para ambos os índices 
de ações a suposição de normalidade pode ser bastante restritiva e muito questionável, mesmo quando se analisa um período extenso de tempo.

Sendo a suposição de normalidade dos retornos base para a formulação de diversos modelos utilizados em finanças, chama a atenção a possível perda informacional quando da utilização dos mesmos. Alguns autores têm desenvolvido uma vertente de pesquisas onde se procura analisar a viabilidade dos modelos quando se relaxa essa pressuposição (COCHRANE, 2005) ou quando se adotam explicitamente outras distribuições teóricas como aproximação para a distribuição empírica dos dados (HAMADA; VALDEZ, 2004). No caso de mercados emergentes, a exemplo do Brasil, a utilização de modelos que se baseiam em pressupostos acerca das distribuições deve ser tratada com cautela ainda maior, visto que os retornos no período analisado apresentaram desvios em relação a normal no terceiro e quarto momentos da distribuição.

As evidências apresentadas pelos dados parecem indicar que trabalhos futuros envolvendo abordagens econométricas no desenvolvimento de modelos financeiros devem evitar pressuposições em relação à distribuição dos retornos de ações, sendo esse um campo promissor de estudo.

\section{REFERÊNCIAS}

BADRINATH, S. G.; CHATTERJEE, S. On measuring skewness and elongation in common stock return distributions: the case of the market index. The Journal of Business, v. 6I, n. 4, p. 451-472, I988. BLACK, F. Capital market equilibrium with restricted borrowing. The Journal of Business, v. 45, n. 3, p. 444-455, I972.

BLAKE, D. Efficiency, risk Aversion and portfolio insurance: an analysis of financial asset portfolios Held by investors in the United Kingdom. The Economic Journal, v. Io6, p. II75-II92, I996.

BOOKSTABER, R. M.; MCDONALD, J. B. A general distribution for describing security price returns. Journal of Business, v. 60, n. 3, p. 40I-424, I987.

BRANCO, M. D’E.; ARELLANO-VALLE, R. Distribuições elípticas assimétricas. São Paulo: Associação Brasileira de Estatística, $\mathrm{I}^{\circ}$ Sinape -26 a 30.7.2004.

BROWN, S. J., WARNER, J. B. Using daily stock returns: the case of event studies. Journal of Financial Economics, v. I4, issue I, I985.

CAMPBELL, J. Y.; LO, A. W.; MACKINLAY, A. C. The econometrics of financial markets. New Jersey: Princeton, I997.

COCHRANE, J. H. Asset pricing. Revised ed. Princeton, NJ: Princeton, 2005.

FAMA, E. F. The behavior of stock market prices. Journal of Business, v. 38, p. 34-105, 1965.

FISHER, R. A. On the absolute criterion for fitting frequency curves. Messenger of Mathematics, v. 4I, p. I55-I60, I9I2. Republicado em Statistical Science, v. I2, n. I, p. 39-4I, I997.

GOLLIER, C. The economics of risk and time. Cambridge, MA: MIT, $200 \mathrm{I}$. 
GOURIEROUX, C.; MONFORT, A. Statistics and econometric models. New York: Cambridge, I995. 2 v. GROSSMAN, S. J.; STIGLITZ, J. E. On the impossibility of informationally efficient markets. The American Economic Review, v. 70, p. 393-408, i980.

GUPTA, A. K.; VARGA, T. Elliptically contoured models in statistics. Holanda, Dordrecht: Kluwer, I993. HAMADA, M.; VALDEZ, E. A. CAPM and option pricing with elliptical distributions. Sidney: University of Technology, Quantitative Finance Research Centre, Fev. 2004. Research Paper I2O.

INGERSOLL JUNIOR, J. E. Theory of financial decision making. Savage, MD: Rowman \& Littlefield, I987.

LINTNER, J. The valuation of risk assets and the selection of risky investments in stock portfolios and capital budgets. Review of Economics and Statistics, v. 47, n. I, p. 13-37, 1965.

MITTELHAMMER, R. C.; JUDGE, G. G.; MILLER, D. J. Econometric foundations. Cambridge, UK: Cambridge, 2000.

ROSENZWEIGH, M. R.; BINSWANGER, H. P. Wealth, weather risk and the composition and profitability of agricultural investments. The Economic Journal, v. I03, p. 56-78, I993.

SHARPE, W. F. Capital asset market prices: a theory of market equilibrium under conditions of risk. The Journal of Finance, v. I9, n. 3, p. 425-442, 1964.

SPANOS, A. Probability theory and statistical inference. Cambridge, UK: Cambridge, I999.

TORRES, R.; BONOMO, M.; FERNANDES, C. A aleatoriedade do passeio na Bovespa: testando a eficiência do mercado acionário brasileiro. Ensaios econômicos EPGE, n. 402, 2000. 